\title{
Improving Anti-Protein-Fouling Property of Polyacrylonitrile Ultrafiltration Membrane by Grafting Sulfobetaine Zwitterions
}

\author{
Hong Meng, Qiang Cheng, Haizhi Wang, and Chunxi Li \\ College of Chemical Engineering, Beijing University of Chemical Technology, Beijing 100029, China \\ Correspondence should be addressed to Hong Meng; menghong@mail.buct.edu.cn
}

Received 29 November 2013; Accepted 3 February 2014; Published 10 March 2014

Academic Editor: Marinos Pitsikalis

Copyright ( 2014 Hong Meng et al. This is an open access article distributed under the Creative Commons Attribution License, which permits unrestricted use, distribution, and reproduction in any medium, provided the original work is properly cited.

\begin{abstract}
Zwitterions show great superiority in the field of polymer membrane surface functionalization, as the synthesis process is simple, the adaptability of functional groups is strong, and zwitterions with strong hydration capacity in aqueous solutions can inhibit protein adsorption. In this study, a polyacrylonitrile ultrafiltration membrane was modified to improve anti-proteinfouling capacity by grafting short-chain sulfonic type zwitterions. 3-Dimethylaminopropylamine was first grafted onto hydrolyzed polyacrylonitrile (PAN) membrane by the activation of 1-(3-dimethylaminopropyl)-3-ethylcarbodiimide hydrochloride (EDC). Subsequently, sulfobetaine zwitterions emerged on the membrane surface by quaternization of 1,3-propane sultone. The sulfobetaine zwitterionic membranes were analyzed for surface chemical composition, hydrophilic properties, and surface and cross-sectional structure of the membrane, by a combination of Fourier transform infrared spectroscopy, X-ray photoelectron spectroscopy, contact angle measurement, and scanning electron microscopy. Static protein adsorption and dynamic filtration experiments were undertaken to show that the modified membrane had excellent resistance to protein adsorption. It was found that the molecular weight cutoff of the substrate membrane had great influence on the flux recovery rate of the modified membrane.
\end{abstract}

\section{Introduction}

Ultrafiltration technology is an environmentally friendly separation method, with unique advantages for the field of biomedicine. Membrane fouling, which results in reduction of separation efficiency, shortening of device life, and increase in cost [1], constitutes a bottleneck in the continuing development of the technology. Therefore, improvement of the anti-protein-fouling surface has become one of the most important research topics in the field of membrane separation [2].

Anti-protein-fouling materials are typically one of two types: polyethylene glycol or zwitterions. Polyethylene glycol is, in most biochemical solutions, readily oxidisable, and high temperatures greatly reduce the antiprotein adsorption, so the antifouling property is not maintained over a long time [3]. Many scholars have begun to theoretically study zwitterions for anti-protein fouling. The protein adsorption resistance of zwitterions is related to the presence of a dense, hydrated layer. Zwitterions have strong hydration capacity by both ion solvation and hydrogen bonding and form a hydrated layer. This is physical structure and energy barrier; the proteins have to destroy the hydration layer by consuming energy to pass the membrane [3-5]. Anti-protein fouling of zwitterions is also associated with the uniformity of the distribution of charge and the electrical neutrality. Charge uniformity, close arrangement, small size, and polarity are necessary for anti-protein fouling on the molecular level [6]. Charge uniformity not only can maximize the hydration ability of the zwitterion but also can reduce the electrostatic interaction between membrane surface and protein [5]. In addition, water molecules, zwitterionic structure, and protein will affect the anti-protein-fouling property. The larger number of alkyl groups in zwitterions increases the distance between the positive and negative charges, which are conducive to enhance the electrostrictive effect and hydration and strengthen the zwitterionic antiprotein resistance $[7,8]$.

A zwitterion is a compound with both a positive and a negative charge. Typical zwitterions are of the carboxylic acid type, the sulfonic acid type, or the phosphoric acid 


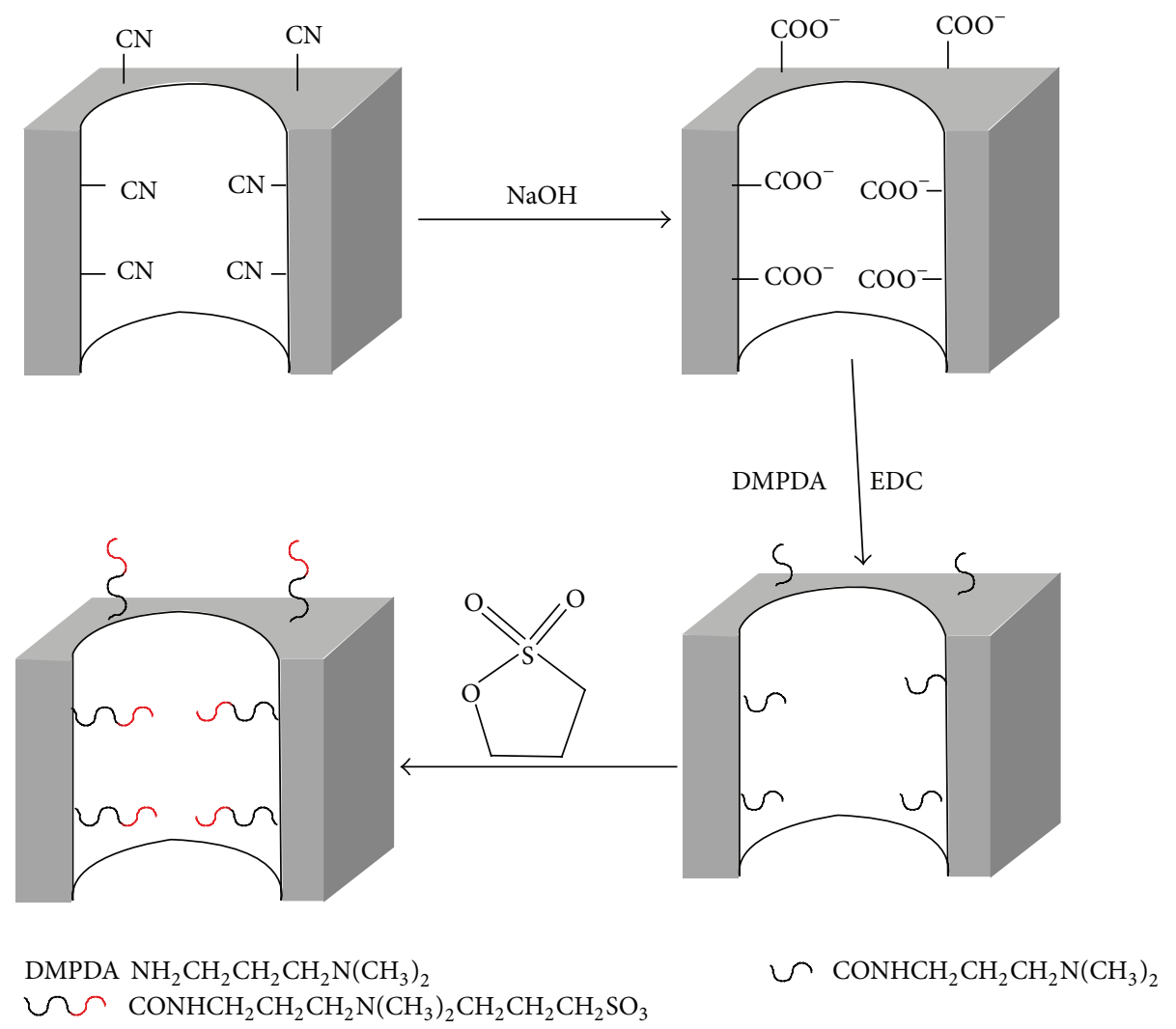

FIGURE 1: Schematic diagram of the surface grafting of zwitterions onto the PAN-based membrane surface.

type. In the past two decades, sulfonic acid and phosphoric acid type zwitterions have been applied to the surface of the polymer membrane in functional modification, for example, 2-methacryloyloxyethyl phosphorylcholine (MPC) or sulfobetaine methacrylate (SBMA). Singer and Nicolson [9] proposed the phospholipid double biomembrane structure in 1972, which showed that similar phospholipids (PC) structure can be used as the antiprotein adsorption surface. PC zwitterionic monomers are difficult to synthesize, and the single functional group limits their practical application $[8$, $10,11]$. During the past decade, many researchers have found and confirmed that the SB zwitterionic functional group and the PC group are extremely similar in structure and are both electrically neutral. Thus, sulfonic acid zwitterions also have the ability of antiprotein adsorption [12-14].

The aim of our current study was to graft short-chain sulfonic type zwitterions to the membrane surface to improve the antifouling capacity. The short-chain molecules could reach and modify membrane surface and membrane pores and have high grafting density. 3-Dimethylaminopropylamine was first grafted onto the hydrolyzed polyacrylonitrile (PAN) membrane by the activation of 1-(3dimethylaminopropyl)-3-ethylcarbodiimide hydrochloride (EDC). Subsequently, sulfonic zwitterions were grafted onto the membrane surface by the quaternization of 1,3-propane sultone. The antifouling property was evaluated by static protein adsorption and dynamic ultrafiltration of protein solution.

\section{Experiment}

2.1. Materials. 1-(3-Dimethylaminopropyl)-3-ethylcarbodiimide hydrochloride (EDC), 1,3-propane sultone, and 3dimethylaminopropylamine were purchased from Shanghai Aladdin Co. (Shanghai, China). Bovine serum albumin (BSA) was obtained from Aobo Star Biotechnology Co., and the PAN-50 (MWCO, 20000) and PAN-350 (MWCO, 150000) membrane was purchased from Sepro Co. Ethanol, hydrofuran (THF), sodium hydroxide, potassium dihydrogen phosphate, disodium phosphate dodecahydrate, sodium chloride, and all other reagents were purchased from the Beijing Chemical Reagent Company and used as received.

2.2. Preparation of the Modified Membrane. Figure 1 shows a schematic diagram of the grafting of zwitterions to the membrane surface. PAN-50 and PAN-350 virgin membranes were hydrolyzed with $1 \mathrm{~mol} / \mathrm{L} \mathrm{NaOH}$ solution at $60^{\circ} \mathrm{C}$. The membrane was rinsed with deionized water for a while. Then, hydrolyzed PAN membrane was soaked in $10 \mathrm{~g} / \mathrm{L}$ EDC buffer solution to be activated for $4 \mathrm{~h}$. After preparing $50 \mathrm{~mL}$ 3-dimethylaminopropylamine solution (the modifier) at concentrations of $2 \mathrm{~g} / \mathrm{L}, 5 \mathrm{~g} / \mathrm{L}$, and $10 \mathrm{~g} / \mathrm{L}$, the hydrolyzed PAN membranes reacted at room temperature for $24 \mathrm{~h}$. The PAN-50 and PAN-350 membranes, which were modified by the $2 \mathrm{~g} / \mathrm{L}$ modifier, are referred to as PAN-50-M1 and PAN350-M1, respectively. Likewise, the membranes modified with 
$5 \mathrm{~g} / \mathrm{L}$ modifier are referred to as PAN-50-M2 and PAN$350-\mathrm{M} 2$, and those modified with the $10 \mathrm{~g} / \mathrm{L}$ modifier are referred to as PAN-50-M3 and PAN-350-M3. After completion of the reaction, the membranes were cleaned to remove impurities. The grafted membrane was placed into the reaction vessel containing $2 \mathrm{~g}$ of 1,3-propane sultone in $50 \mathrm{~mL}$ tetrahydrofuran (THF). The reaction lasted $24 \mathrm{~h}$ at $50^{\circ} \mathrm{C}$; then the membrane was washed to remove impurities from the surface.

2.3. Characterization of Modified Membranes. The surface chemical composition of the membranes was analyzed by X-ray photoelectron spectroscopy (XPS) (ESCALAB 250, USA), using $\mathrm{Al}$ as the radiation source. Fourier transform infrared (FTIR, Nicolet-560, Shimadzu Corporation) spectroscopy showed functional groups over the scanning range of $4000 \mathrm{~cm}^{-1}$ to $400 \mathrm{~cm}^{-1}$. The surface and cross-section morphologies of the modified ultrafiltration membranes were observed by scanning electron microscopy (SEM) (S-4700, Hitachi). The static contact angle of membranes was measured at room temperature using a contact angle goniometer (JC2000C Contact Angle Meter, Powereach Co., Shanghai, China).

2.4. Static Protein Adsorption Experiments. PAN modified membranes were cut into a circle and put into a tube containing $10.0 \mathrm{~mL}$ of $1 \mathrm{mg} / \mathrm{mL}$ BSA aqueous solution at $30 \pm$ $1^{\circ} \mathrm{C}$. After incubation for $6 \mathrm{~h}$, protein concentrations in the solutions were analyzed with a UV-vis spectrophotometer (Labtec UV, China), and the amount of BSA adsorbed onto the membranes was calculated.

2.5. Dynamic Ultrafiltration Experiments. The antifouling properties under dynamic flow conditions were evaluated via protein ultrafiltration experiments at ambient temperature. A schematic diagram is shown in Figure 2. A dead-end stirred cell with effective membrane area $38.5 \mathrm{~cm}^{2}$ was used for the ultrafiltration study. All ultrafiltration experiments were carried out with continuous stirring at $25 \pm 1^{\circ} \mathrm{C}$. Each membrane was initially compacted with deionized water for $30 \mathrm{~min}$ at $140 \mathrm{kPa}$. The pressure was then lowered to the operating pressure of $100 \mathrm{kPa}$; the water flux $(J)$ and retention rate $(R)$ were calculated by the following equation:

$$
\begin{gathered}
J=\frac{V}{A \Delta t}, \\
R=\left(1-\frac{C_{p}}{C_{f}}\right) \times 100 \%,
\end{gathered}
$$

where $V$ is the volume of permeated water $(\mathrm{L}), A$ is the membrane area $\left(\mathrm{m}^{2}\right)$, and $\Delta t$ is the permeation time (h). In this case, the measured water flux is referred to as $J_{w 1}$. After switching the feed solution to $1.0 \mathrm{mg} / \mathrm{mL}$ BSA solution, BSA filtration was conducted for $40 \mathrm{~min}$, and the flux $\left(J_{p}\right)$ was measured. Finally, the membrane was cleaned with deionized water for $30 \mathrm{~min}$, and the pure water flux $\left(J_{w 2}\right)$ was measured.

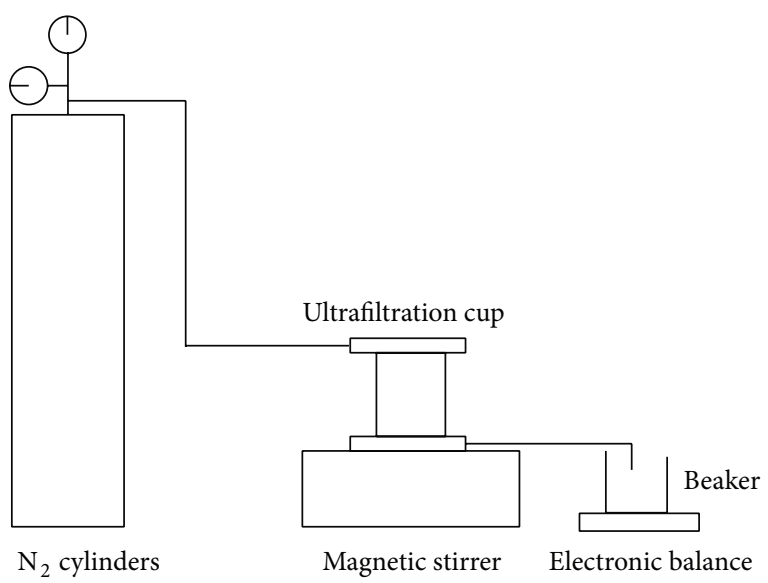

FIGURE 2: Schematic diagram of ultrafiltration device.

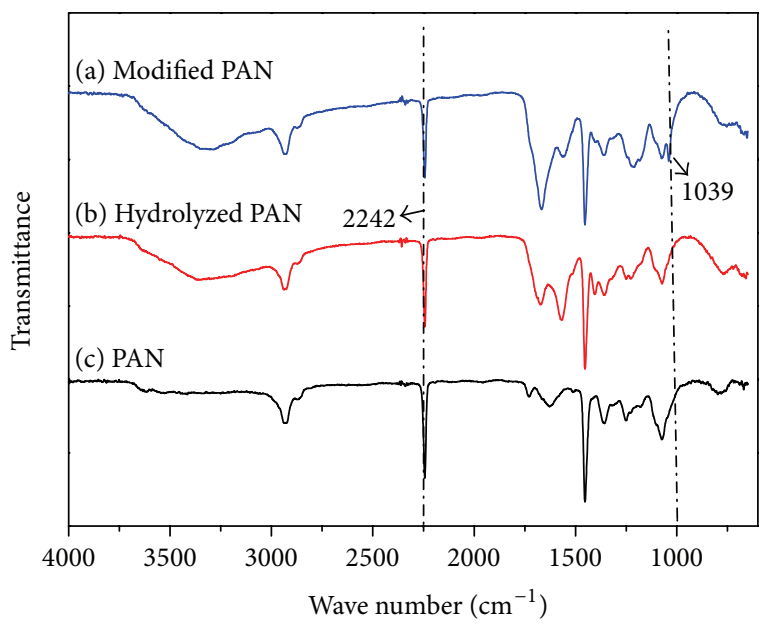

FIGURE 3: FTIR spectra of PAN membrane: (a) the modified membrane, (b) the hydrolyzed membrane, and (c) the virgin PAN membrane.

Flux recovery rate $\left(R_{\mathrm{FR}}\right)$ represents membrane fouling index

$$
R_{\mathrm{FR}}=\frac{J_{w 2}}{J_{w 1}} .
$$

\section{Results and Discussion}

3.1. FTIR and XPS Analyses. The chemical composition of modified membrane was characterized by FTIR spectroscopy; the spectra for PAN membrane are shown in Figure 3. PAN-50 and PAN-350 membrane had similar IR spectrum. The characteristic absorption band of $-\mathrm{CN}$ was present at $2242 \mathrm{~cm}^{-1}$, and the sulfonic acid group of symmetric and asymmetric stretching vibration absorption peaks was observed at $1039 \mathrm{~cm}^{-1}$, indicating the successful grafting of zwitterions onto the surface of the membrane (see Figure 4).

The chemical composition of the membrane surface was also characterized by XPS, by analyzing the change of 

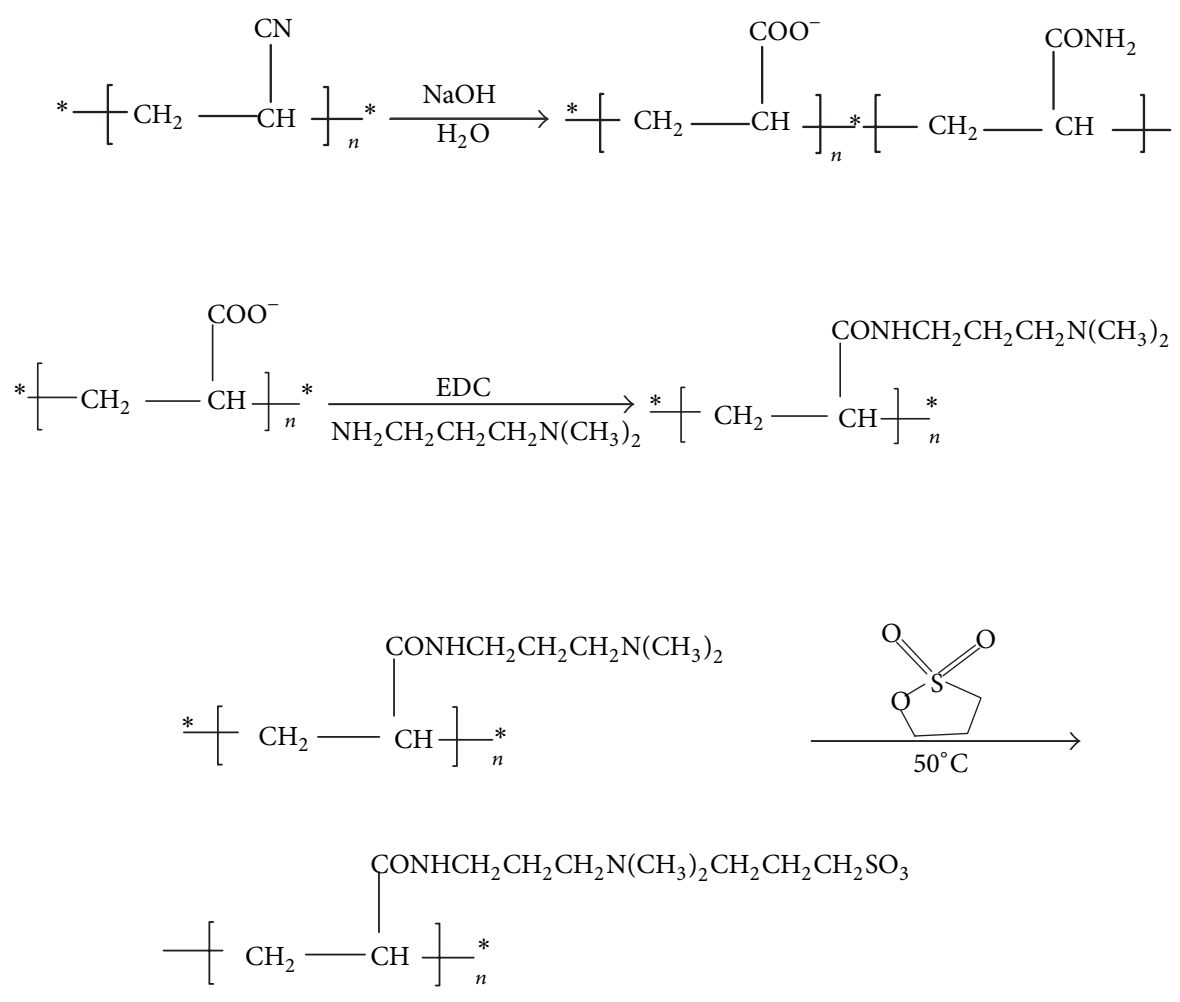

Figure 4

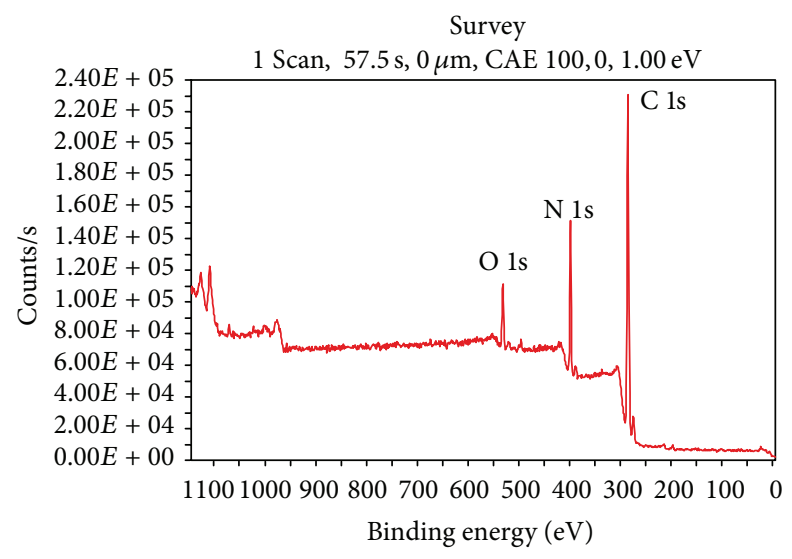

(a)

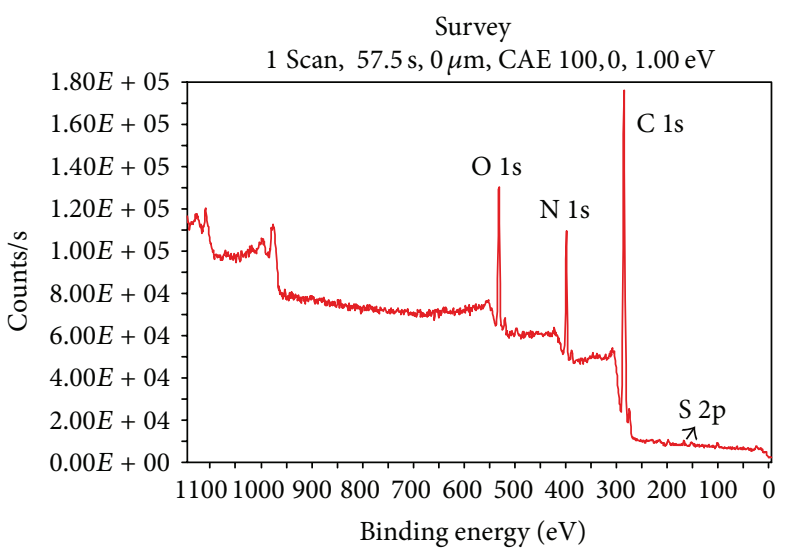

(b)

FIgURE 5: XPS spectra of (a) virgin PAN-350 membrane and (b) PAN-350-M3 membrane.

elements on membrane surface at various steps of the grafting process. As can be seen in Table 1 (PAN-50), the $-\mathrm{CN}$ of the PAN blank membrane generated $-\mathrm{COO}^{-}$by alkaline hydrolysis, leading to an increase in O from $10.9 \%$ to $13.96 \%$ and a decrease of $\mathrm{N}$ from $14.69 \%$ to $12.14 \%$. The $\mathrm{N}$ content of the PAN-50 zwitterionic modified membrane increased by different degrees, from $13.12 \%$ to $13.8 \%$, depending on grafting concentration, caused by the $-\mathrm{NH}$ bond generated by the reaction between $-\mathrm{COO}^{-}$from the membrane surface and the quaternary ammonium bond by quaternarization. In addition, $S$ content was detected for the PAN membrane modified with the SB zwitterion, which suggested that the sulfobetaine zwitterion was successfully grafted onto the PAN membrane. Moreover, the $S$ content also increased from $0.54 \%$ to $0.97 \%$ with increasing grafting solution concentration. It was observed from the element percentage change that the sulfonic zwitterionic group was successfully grafted onto the PAN-50 membrane. PAN-350 had a similar pattern, which was shown in Table 2 and Figure 5. After hydrolyzing, $\mathrm{O}$ increased from $8.6 \%$ to $11.03 \%$, and $\mathrm{N}$ decreased from $14.28 \%$ to $13.32 \%$. The S content appeared and increased, to some degree, by further modification. $S$ content increased from $0.46 \%$ to $0.52 \%$ when the modifier concentration changed from $2 \mathrm{~g} / \mathrm{L}$ to $10 \mathrm{~g} / \mathrm{L}$. 


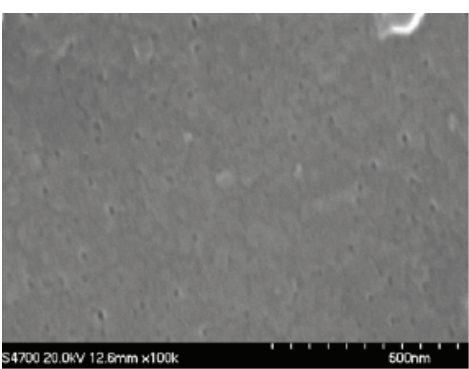

(a)

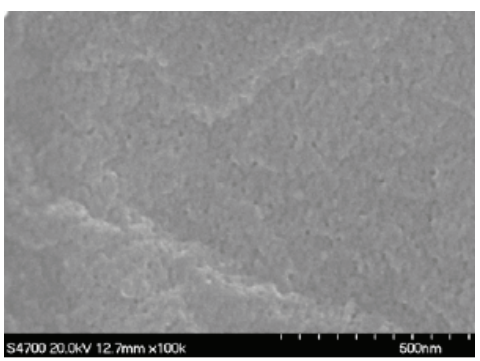

(d)

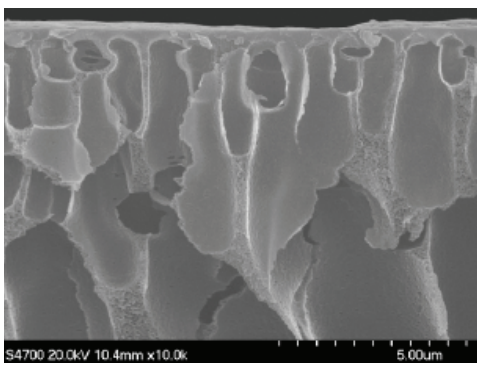

$(\mathrm{g})$

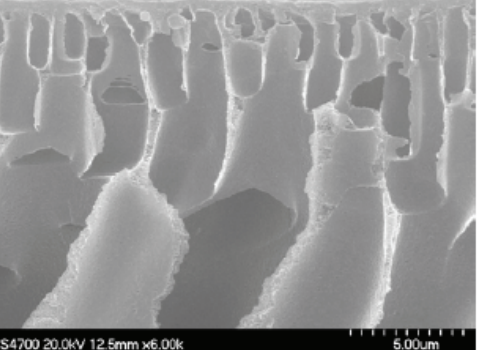

(b)

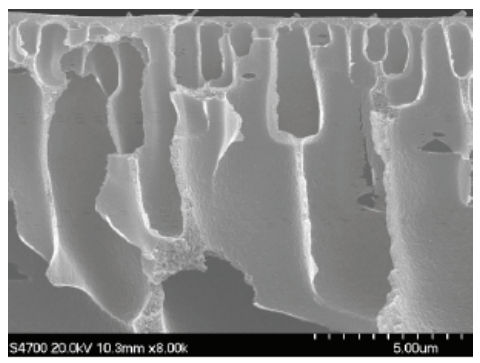

(e)

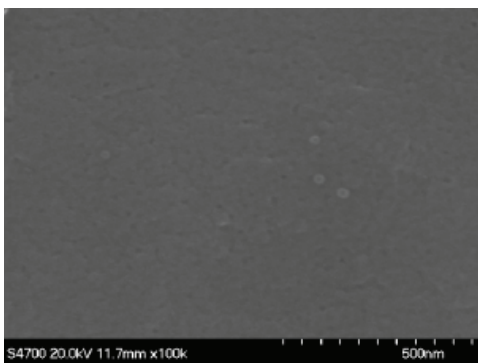

(h)

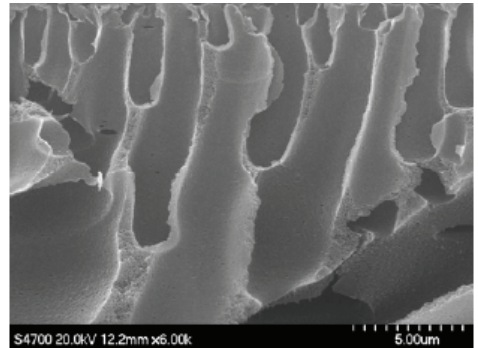

(c)

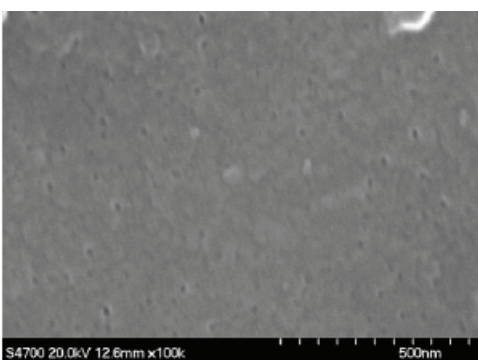

(f)

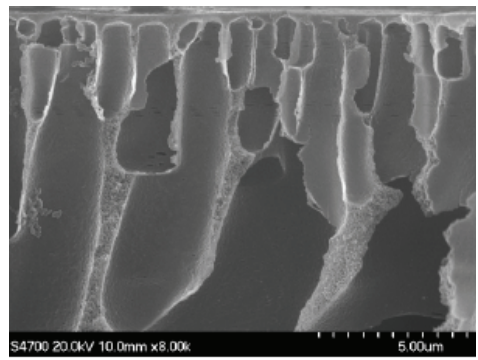

(i)

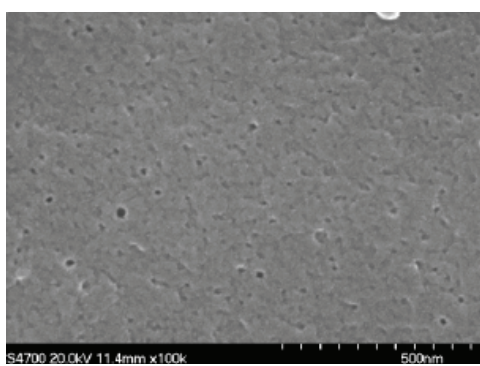

(j)

Figure 6: Sectional structure and surface structure of PAN-50 membrane. (a) and (b) PAN, (c) and (d) hydrolyzed PAN, (e) and (f) PAN-M1, (g) and (h) PAN-M2, and (i) and (j) PAN-M3.

TABLE 1: The date of XPS of PAN-50 membrane.

\begin{tabular}{lcccc}
\hline & $\mathrm{C}$ & $\mathrm{O}$ & $\mathrm{N}$ & $\mathrm{S}$ \\
\hline PAN-50 & 74.14 & 10.9 & 14.69 & 0 \\
Hydrolyzed PAN-50 & 75.71 & 13.96 & 12.14 & 0 \\
PAN-50-M1 & 74.57 & 11.77 & 13.12 & 0.54 \\
PAN-50-M2 & 75.5 & 11.47 & 12.37 & 0.66 \\
PAN-50-M3 & 75 & 10.23 & 13.8 & 0.97 \\
\hline
\end{tabular}

Examination of the impact of modification on the membrane surface and the internal structure was carried out via
TABLE 2: The date of XPS of PAN-350 membrane.

\begin{tabular}{lcccc}
\hline & $\mathrm{C}$ & $\mathrm{O}$ & $\mathrm{N}$ & $\mathrm{S}$ \\
\hline PAN-350 & 76.85 & 8.6 & 14.55 & 0 \\
Hydrolyzed PAN-350 & 75.66 & 11.03 & 13.32 & 0 \\
PAN-350-M1 & 74.36 & 11.61 & 13.57 & 0.46 \\
PAN-350-M2 & 74.24 & 11.89 & 13.41 & 0.47 \\
PAN-350-M3 & 72.98 & 11.79 & 14.72 & 0.52 \\
\hline
\end{tabular}

SEM. As can be seen in Figures 6 and 7, the membrane structure contained a dense epidermal layer and a porous 


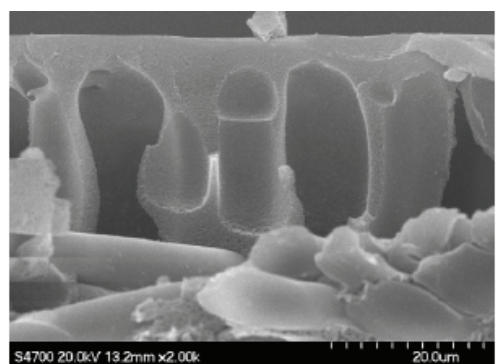

(a)

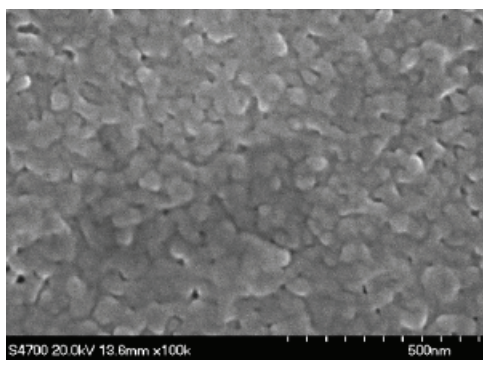

(d)

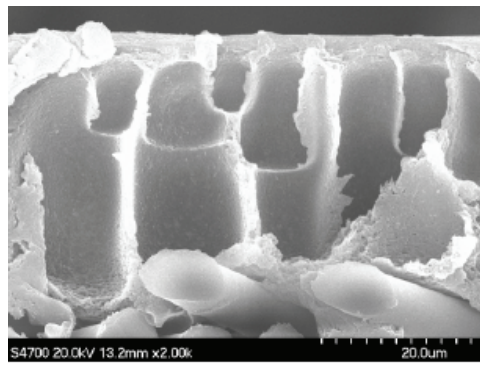

(g)

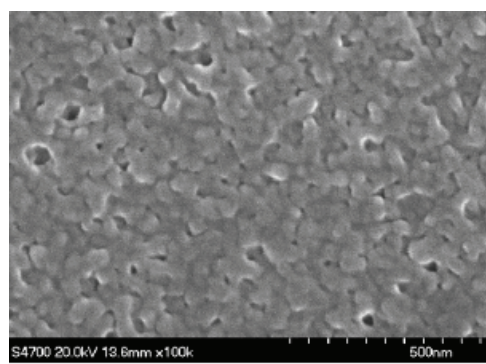

(b)

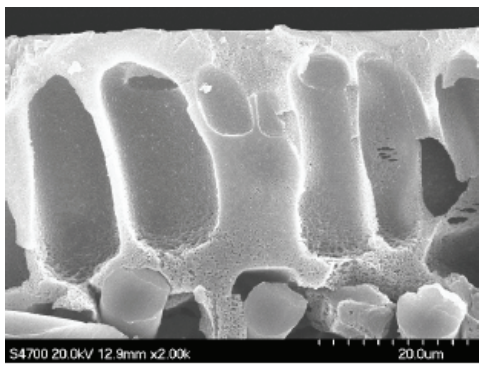

(e)

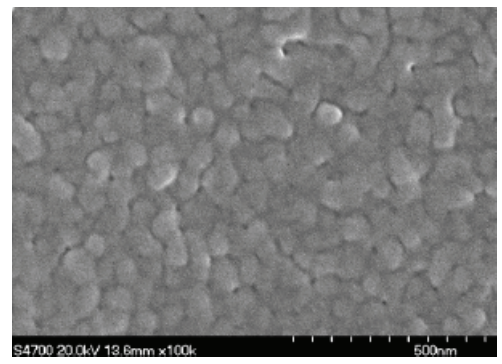

(h)

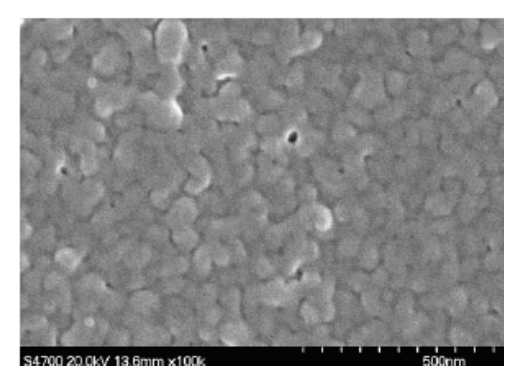

(j)

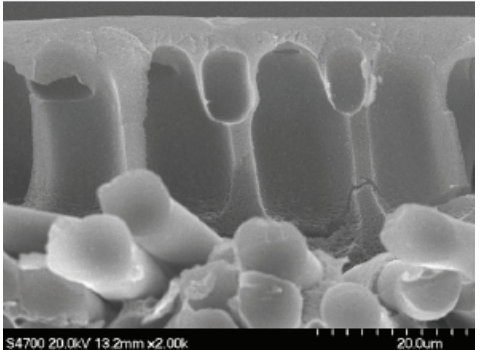

(c)

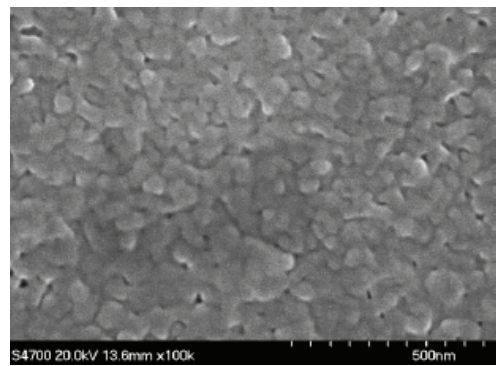

(f)

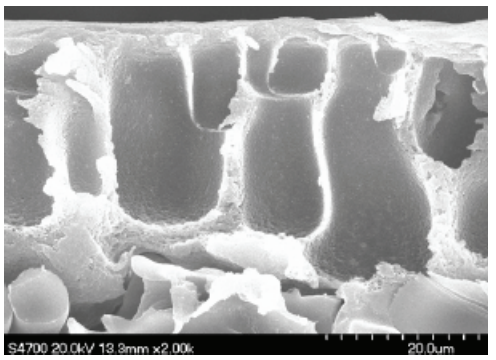

(i)

Figure 7: Sectional structure and surface structure of PAN-350 membrane. (a) and (b) PAN, (c) and (d) hydrolyzed PAN, (e) and (f) PAN-M1, (g) and (h) PAN-M2, and (i) and (j) PAN-M3.

support layer. The cross-sectional structure of the PAN membrane was finger-like (Figures 6(a) and 7(a)). The dense epidermal layer played a major factor in protein separation. In order to avoid increasing the mass transfer resistance from support layer, it was expected that the grafting of shortchain sulfobetaine zwitterions had insignificant effects on the finger-like structure. It can be seen in the figures that the skin layer and the support layer did not change dramatically, indicating that the modified membrane structure was not damaged. Modification only changed the chemical composition of the membrane surface and the membrane pores.
The contact angle of the membrane is considered to be the main performance characteristic of the membrane surface hydrophilicity. As can be seen from Figure 8, the hydrolysis process decreased the contact angle, the PAN50 contact angle decreased from $60^{\circ}$ to $36^{\circ}$, and the PAN350 contact angle decreased from $64^{\circ}$ to $40^{\circ}$. The decrease in contact angle indicates an increase in the hydrophilicity of the PAN membrane, which is due to the formation of hydrophilic carboxylic groups. The contact angle of the PAN50 membrane increased from $36^{\circ}$ for the hydrolyzed case to $51^{\circ}$ for the zwitterionic modified case, and, similarly, 


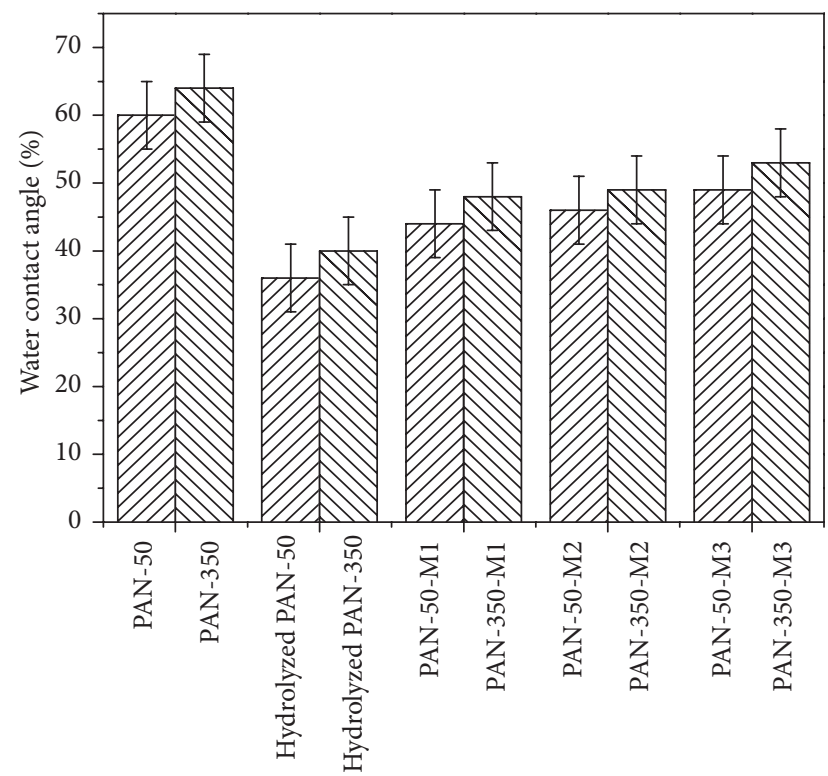

FIGURE 8: Contact angle of PAN membrane.

the PAN-350 contact angle increased from $40^{\circ}$ to $53^{\circ}$. Compared to the short-chain $-\mathrm{COO}^{-}$of the hydrolyzed membrane, the hydrophilicity of long-chain SB zwitterions was relatively poor, so the contact angle increased. However, the zwitterion with positive and negative charges could combine with water and formed a hydrated layer, resisting the contraction between the membrane surface and the protein, and, thus, the zwitterionic membrane had better fouling resistance.

3.2. Static Adsorption and Dynamic Filtration of Protein Solutions. As can be seen from Figure 9, after hydrolysis, the amount of protein adsorption of the PAN-50 membrane decreased to $51 \%$ and that of the PAN-350 membrane decreased to $55 \%$. The membrane surface generated hydrophilic carboxylic groups; carboxyl groups on the membrane surface appeared negatively charged, and BSA appeared negatively charged in the buffer solution with $\mathrm{pH}=$ 7.4. So, both charge repulsion and hydrophilicity reduced the degree of protein adsorption of the hydrolyzed membrane surface. The BSA adsorption of the PAN-50 and PAN-350 zwitterionic modified membranes decreased with increasing concentration of 3-dimethylaminopropylamine in the grafting solution. This is mainly due to the zwitterions on the membrane surface. The more zwitterions grafted onto PAN membrane, the less protein adsorption took place. Zwitterions have strong hydration capacity, by both ion solvation and hydrogen bonding, and form a hydrated layer preventing protein adsorption. Uniform charge maximized the hydration capacity of the zwitterions. Therefore, the zwitterionic modified membrane had better antifouling performance.

In subsequent experiments, dynamic filtration of the protein solution was also conducted to investigate the antiprotein-fouling capacity of PAN-based zwitterionic membranes. The pure water flux of the PAN-50 blank membrane decreased from $551.24 \mathrm{~L} /\left(\mathrm{m}^{2} \cdot \mathrm{h}\right)$ to $374 \mathrm{~L} /\left(\mathrm{m}^{2} \cdot \mathrm{h}\right)$ after hydrolysis and that of the PAN-350 blank membrane decreased

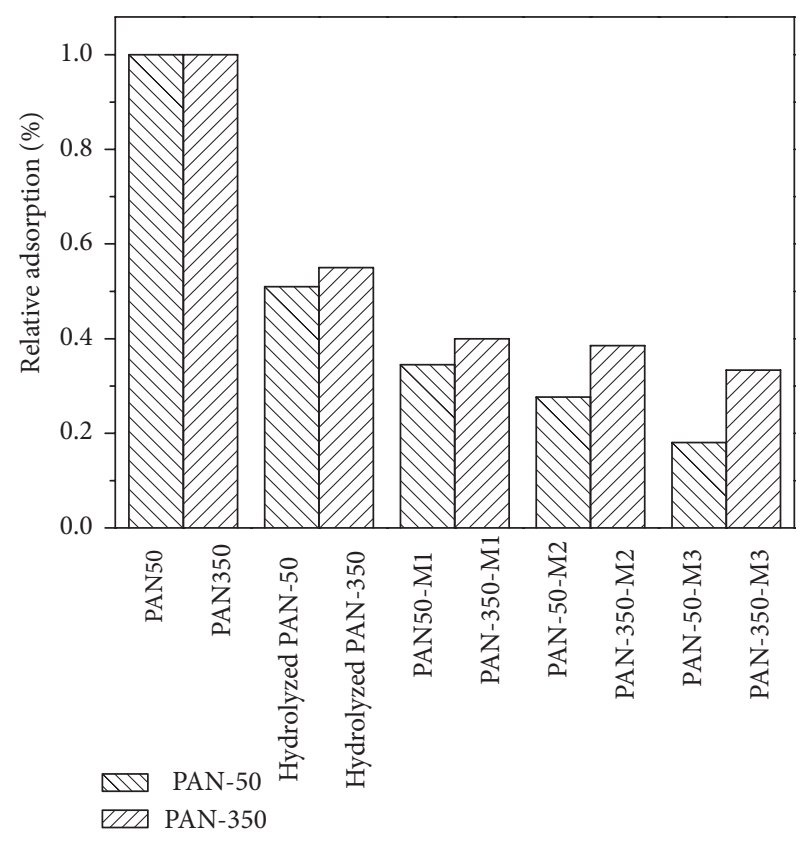

Figure 9: Protein static adsorption data.

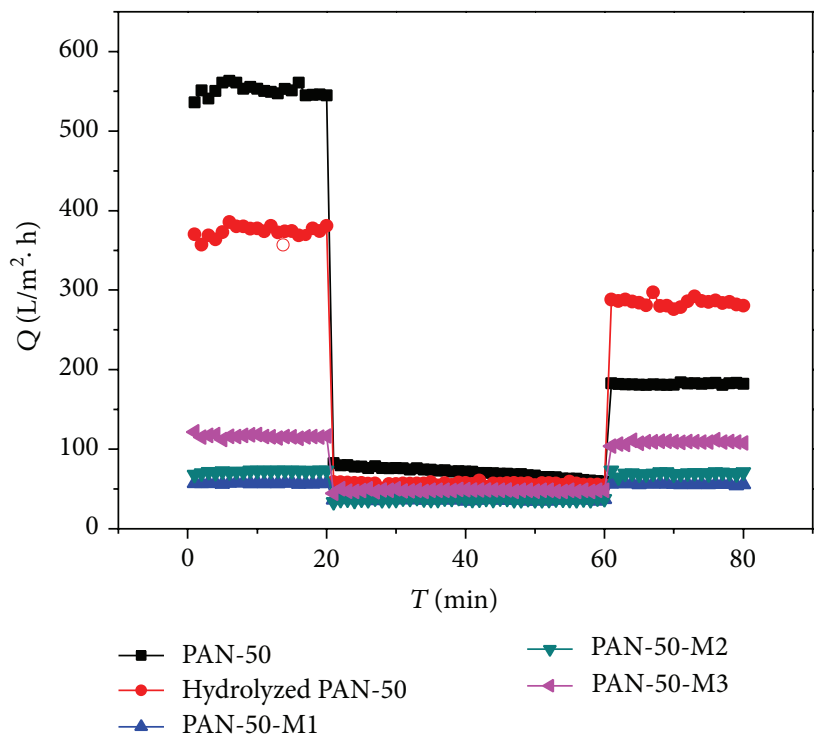

Figure 10: The flux of the PAN-50 modified membrane.

from $740.59 \mathrm{~L} /\left(\mathrm{m}^{2} \cdot \mathrm{h}\right)$ to $423.24 \mathrm{~L} /\left(\mathrm{m}^{2} \cdot \mathrm{h}\right)$. This was because the carboxylic chains of the hydrolyzed PAN membrane easily swelled and segregated on the surface of the membrane, causing narrowing of the membrane pore size. As can be seen in Figure 10, the flux recovery rate of the PAN-50 zwitterionic modified membrane reached more than 98.4\%, which was much higher than that of the blank PAN membrane $(33 \%)$. This was because more zwitterionic groups could combine with water near the surface of the membrane and form a thicker hydration layer. The rejection rate of BSA was maintained at around 99\%, but pure water of the PAN-50 zwitterionic modified membrane was lost largely. 


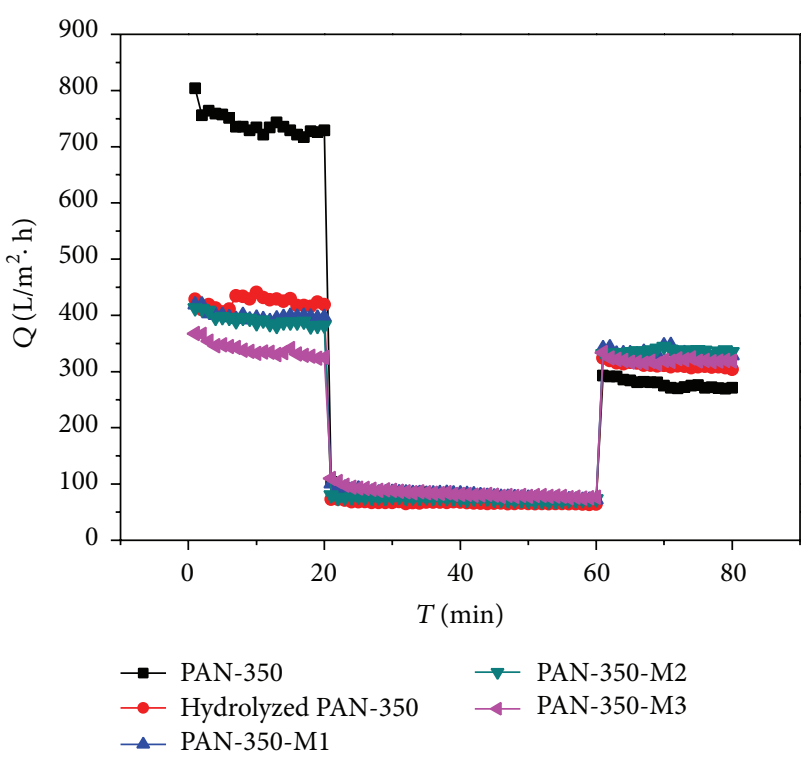

Figure 11: The flux of the PAN-350 modified membrane.

As can be seen in Figure 11, the water flux and protein flux of the PAN-350 zwitterionic modified membrane maintained high water flux. PAN-350 zwitterionic modified membrane reached more than $98.4 \%$, which was much higher than that of the blank PAN membrane (33\%). However, compared with the PAN-50 membrane, the flux loss of zwitterionic modified membrane was less than that of PAN-50 under the same operation conditions; furthermore, the flux recovery rate maintained a high level of more than $83 \%$. The larger MWCO meant larger pore size of the substrate membrane. When the zwitterions grafted onto PAN substrate membrane, the short-chain SB had less influence on the change of pore size of PAN-350 membrane. This indicated that the relatively larger pore membrane also achieved better results.

The reusability of the membrane is an important factor in the separation performance. Figure 12 shows the variation of the flux recovery rate of the PAN-50 membrane over three operation runs. Between cycles, the membrane was washed with pure water for $0.5 \mathrm{~h}$. The figure shows that, after three runs, the flux recovery rate of the PAN-50 zwitterionic modified membrane was $93 \%$, while the flux recovery rate of the PAN-50 unmodified membrane was $22 \%$. The PAN50 zwitterionic modified membrane therefore had good antiprotein-fouling performance.

As can be seen in Figure 13, the PAN-350 modified membrane three-cycle stability test results show that water flux of PAN-350 modified membrane decayed to $77.4 \%$ of the original membrane, while the blank membrane reduced to $22 \%$ of the original membrane. The water flux of the unmodified PAN-350 reduced to $28.8 \%$ of the original membrane, while modified PAN-350 membrane only decayed to $77.4 \%$. These results show that the modified PAN-350 membrane also effectively reduced the irreversible fouling effects, with good flux recovery performance.

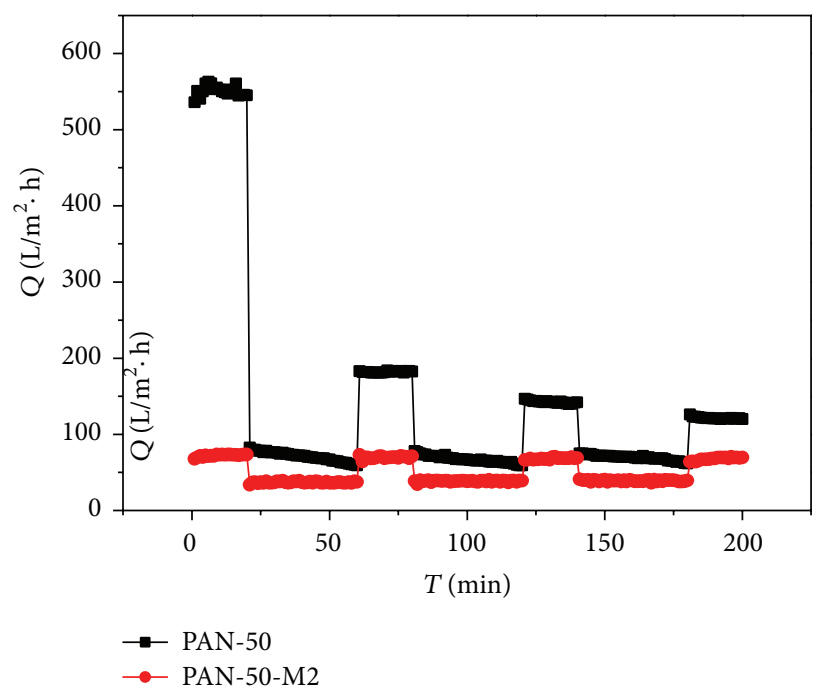

FIgure 12: The three-cycle flux data for the PAN-50 membrane.

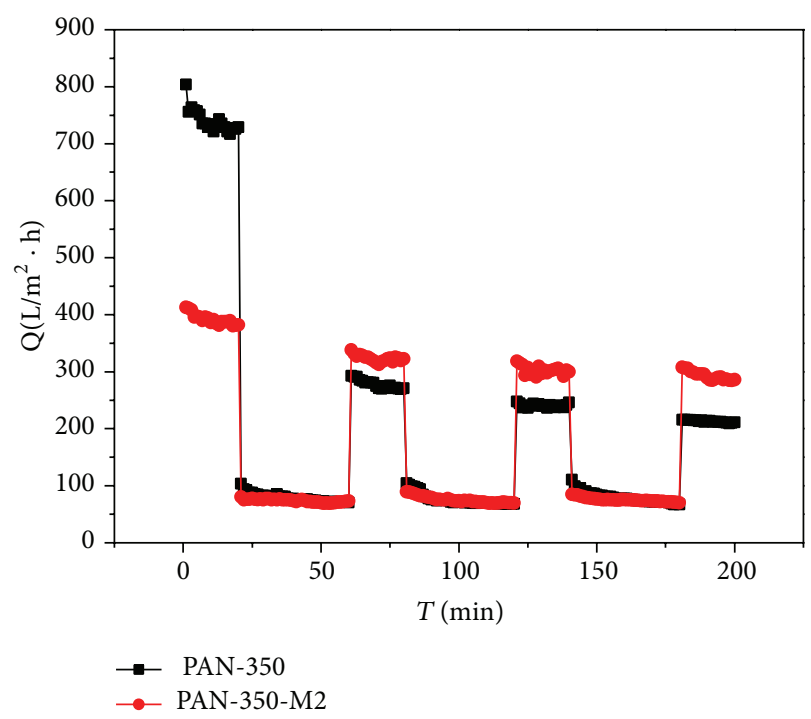

Figure 13: The three-cycle flux data for the PAN-350 membrane.

\section{Conclusions}

An approach to construct protein fouling ultrafiltration membranes surface by grafting short-chain sulfobetaine zwitterionic molecules as modifiers has been presented. Grafting concentration had no obvious effect on the water flux of the PAN-350 membrane, which had larger pores, but significantly affected the water flux of the PAN-50 membrane with smaller pores. Protein static adsorption and dynamic filtration experiments showed that the modified membrane had excellent resistance to protein adsorption and good antiprotein-fouling capacity. This is because zwitterions form a hydrated layer by both ion solvation and hydrogen bonding, preventing protein adsorption. Uniform charge distribution maximized the hydrated capacity of the zwitterions. It is concluded that zwitterions can play a crucial role in membrane surface anti-protein fouling. 


\section{Symbols and Abbreviations}

Symbols

PAN-50-M1: Modified PAN-50 membranes prepared using $2 \mathrm{~g} / \mathrm{L} 3$-dimethylaminopropylamine

PAN-50-M2: Modified PAN-50 membranes prepared using $5 \mathrm{~g} / \mathrm{L}$ 3-dimethylaminopropylamine

PAN-50-M3: Modified PAN-350 membranes prepared using $10 \mathrm{~g} / \mathrm{L}$ 3-dimethylaminopropylamine

PAN-350-M1: Modified PAN-350 membranes prepared using $2 \mathrm{~g} / \mathrm{L}$ 3-dimethylaminopropylamine

PAN-350-M2: Modified PAN-350 membranes prepared using $5 \mathrm{~g} / \mathrm{L} 3$-dimethylaminopropylamine

PAN-350-M3: Modified PAN-350 membranes prepared using $10 \mathrm{~g} / \mathrm{L} 3$-dimethylaminopropylamine

$J_{w}: \quad$ Flux of water

$J_{p}: \quad$ Flux of protein

$R_{\mathrm{FR}}$ : $\quad$ Recovery rate of flux

$R: \quad$ Rejection rate

$C_{p}: \quad$ Penetrating concentration

$C_{f}: \quad$ Retentating concentration

$V:$

A:

$t:$

$J:$

Volume of the permeated solution

Membrane area

Permeation time

Flux.

\section{Abbreviations}

MPC: 2-Methacryloyloxyethyl phosphorylcholine glycol

SBMA: Sulfobetaine methacrylate

MWCO: Molecular weight cutoff

PC: Phospholipids

PAN: Polyacrylonitrile

BSA: Bovine serum albumin

THF: Tetrahydrofuran

EDC: 1-Ethyl-(3-3-dimethylaminopropyl)-

carbodiimide

hydrochloride

SEM: Scanning electron microscopy

FTIR: Fourier transform infrared

XPS: X-ray photoelectron spectroscopy

UV: Ultraviolet.

\section{Conflict of Interests}

The authors declare that there is no conflict of interests regarding the publication of this paper.

\section{Acknowledgments}

The project was supported by the National Natural Science Foundation of China (Program no. 21076010) and the Fundamental Research Funds for the Central Universities (Program no. 221105).

\section{References}

[1] R. Baker, Membrane Technology and Applications, Wiley, 2012.

[2] D. Rana and T. Matsuura, "Surface modifications for antifouling membranes," Chemical Reviews, vol. 110, no. 4, pp. 2448-2471, 2010.

[3] S. Chen, L. Li, C. Zhao, and J. Zheng, "Surface hydration: principles and applications toward low-fouling/nonfouling biomaterials," Polymer, vol. 51, no. 23, pp. 5283-5293, 2010.

[4] E. Ostuni, R. G. Chapman, R. E. Holmlin, S. Takayama, and G. M. Whitesides, "A survey of structure-property relationships of surfaces that resist the adsorption of protein," Langmuir, vol. 17, no. 18, pp. 5605-5620, 2001.

[5] S. Chen, J. Zheng, L. Li, and S. Jiang, "Strong resistance of phosphorylcholine self-assembled monolayers to protein adsorption: insights into nonfouling properties of zwitterionic materials," Journal of the American Chemical Society, vol. 127, no. 41, pp. 14473-14478, 2005.

[6] D. M. Jones, A. A. Brown, and W. T. S. Huck, "Surface-initiated polymerizations in aqueous media: effect of initiator density," Langmuir, vol. 18, no. 4, pp. 1265-1269, 2002.

[7] O. Azzaroni, A. A. Brown, and W. T. Huck, "UCST wetting transitions of polyzwitterionic brushes driven by self-association," Angewandte Chemie, vol. 118, pp. 1802-1806., 2006.

[8] H. Kitano, M. Imai, K. Sudo, and M. Ide, "Hydrogen-bonded network structure of water in aqueous solution of sulfobetaine polymers," Journal of Physical Chemistry B, vol. 106, no. 43, pp. 11391-11396, 2002.

[9] S. J. Singer and G. L. Nicolson, "The fluid mosaic model of the structure of cell membranes," Science, vol. 175, no. 4023, pp. 720731, 1972.

[10] R. G. Laughlin, "Fundamentals of the zwitterionic hydrophilic group," Langmuir, vol. 7, no. 5, pp. 842-847, 1991.

[11] Z.-K. Xu, Q.-W. Dai, J. Wu, X.-J. Huang, and Q. Yang, "Covalent attachment of phospholipid analogous polymers to modify a polymeric membrane surface: a novel approach," Langmuir, vol. 20, no. 4, pp. 1481-1488, 2004.

[12] Y.-C. Chiang, Y. Chang, A. Higuchi, W.-Y. Chen, and R.-C. Ruaan, "Sulfobetaine-grafted poly(vinylidene fluoride) ultrafiltration membranes exhibit excellent antifouling property," Journal of Membrane Science, vol. 339, no. 1-2, pp. 151-159, 2009.

[13] Y.-H. Zhao, K.-H. Wee, and R. Bai, "Highly hydrophilic and low-protein-fouling polypropylene membrane prepared by surface modification with sulfobetaine-based zwitterionic polymer through a combined surface polymerization method," Journal of Membrane Science, vol. 362, no. 1-2, pp. 326-333, 2010.

[14] W. K. Cho, B. Kong, and I. S. Choi, "Highly efficient nonbiofouling coating of zwitterionic polymers: poly((3-(methacryloylamino)propyl)-dimethyl(3-sulfopropyl)ammonium hydroxide)," Langmuir, vol. 23, no. 10, pp. 5678-5682, 2007. 

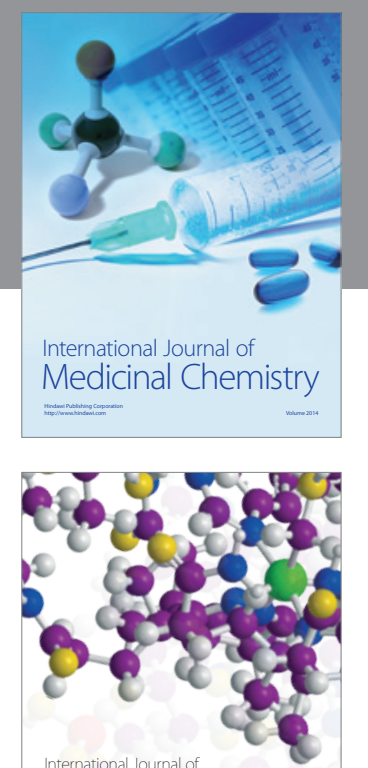

\section{Carbohydrate} Chemistry

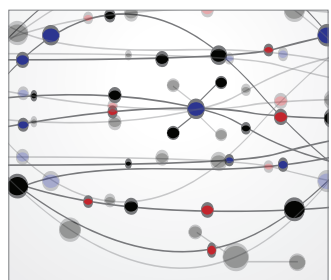

The Scientific World Journal
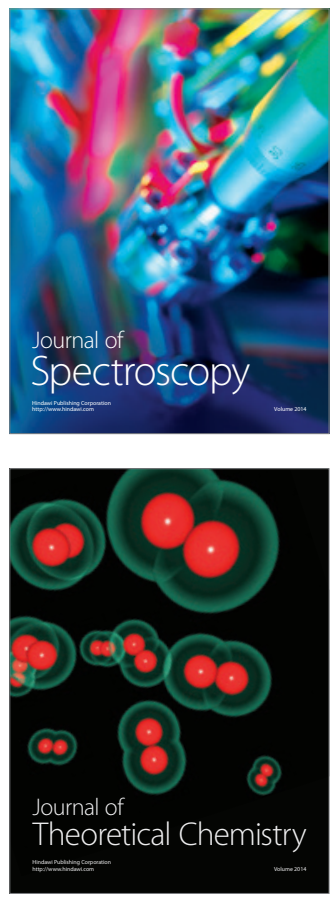
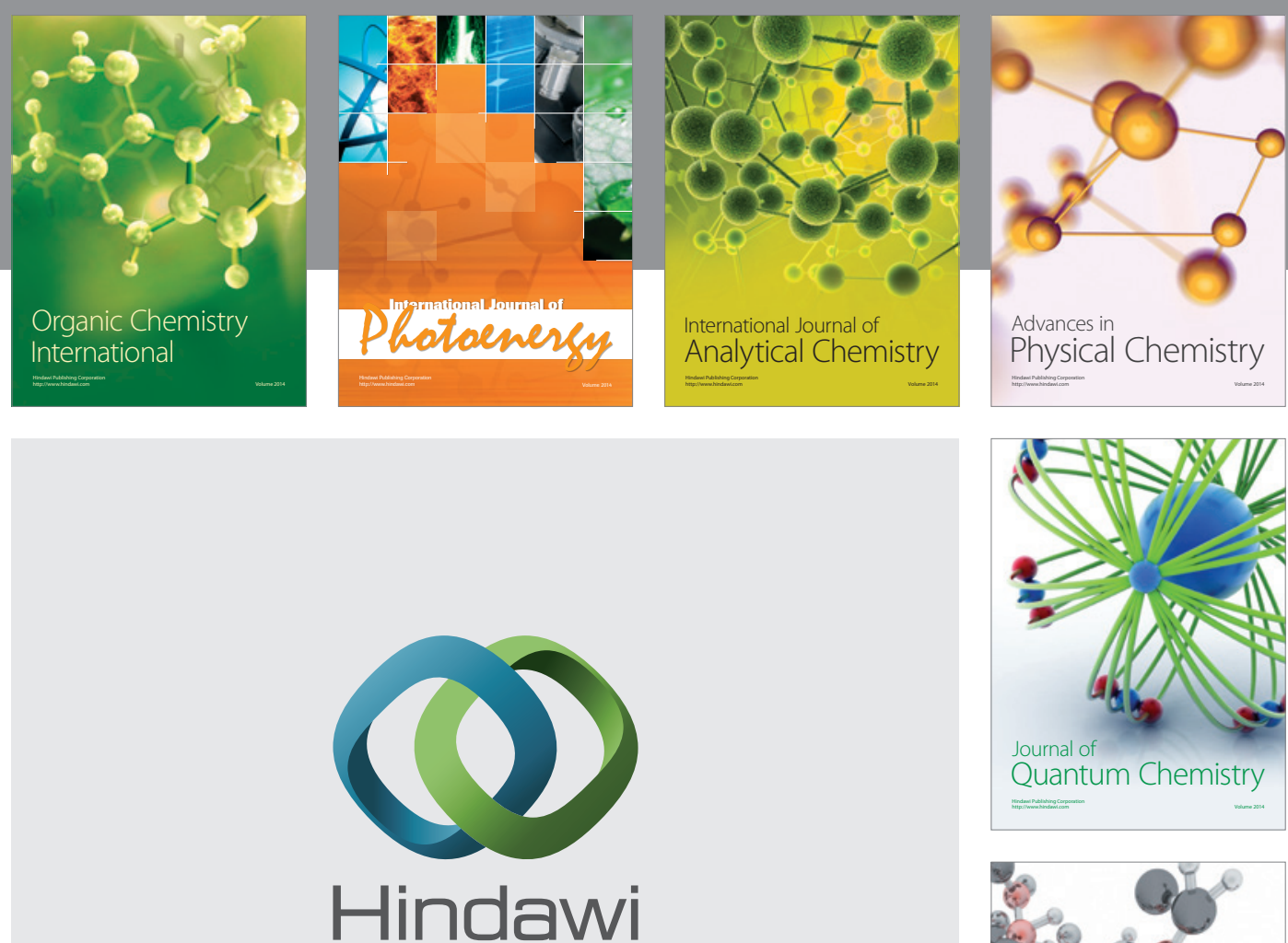

Submit your manuscripts at

http://www.hindawi.com

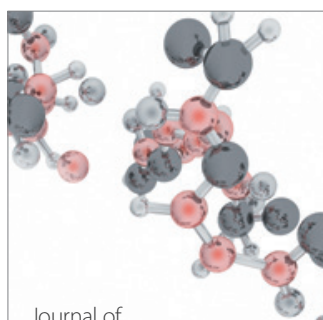

Analytical Methods

in Chemistry

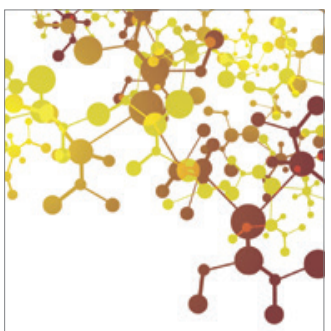

Journal of

Applied Chemistry

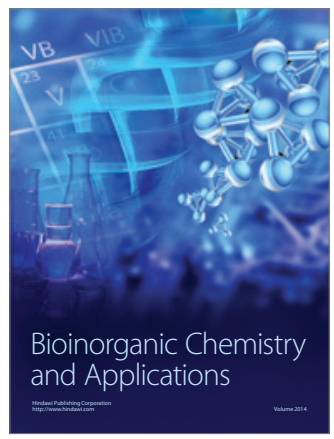

Inorganic Chemistry
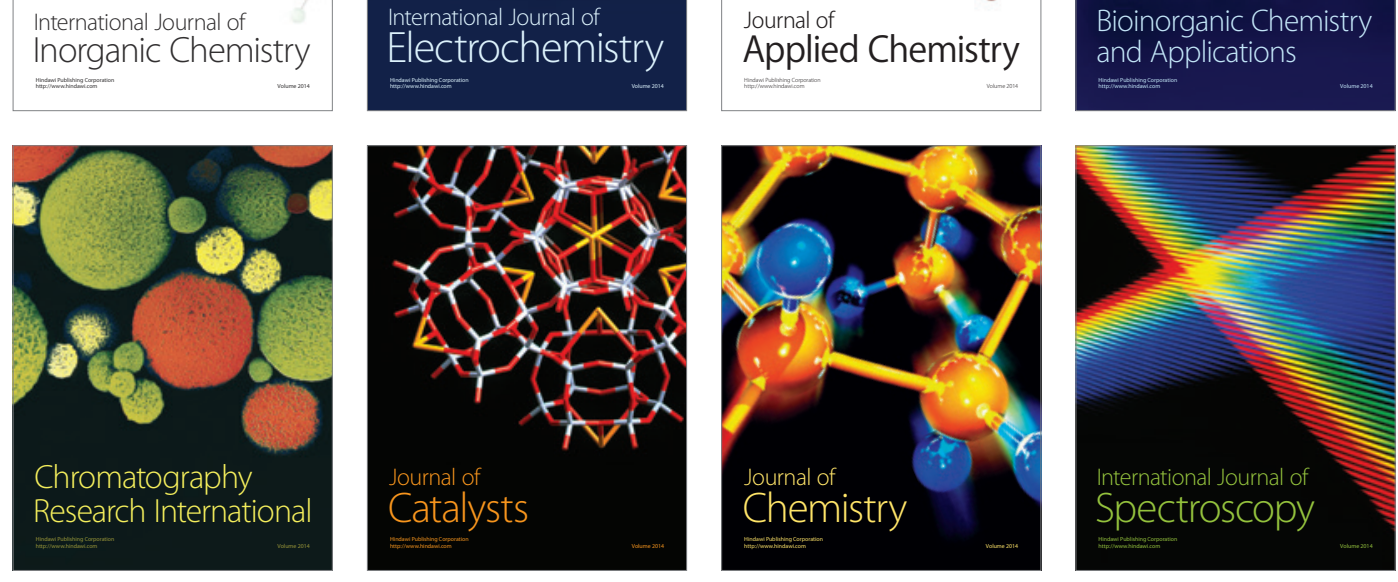